\title{
Comunicación

\section{El mundo de los periodistas: aspectos teóricos y metodológicos}

FABIO HENRIQUE PEREIRA ${ }^{1}$

El artículo se propone discutir la pertinencia de la noción sociológica de "mundo social" aplicada a los estudios de periodismo. Basado en la tradición del interaccionismo simbólico, este concepto es generalmente utilizado para analizar los fenómenos socialmente reconocibles, sin necesidad de estar situados en un espacio institucionalizado. En este caso, se entiende que la comprensión sobre el periodismo no puede limitarse a las prácticas de la producción noticiosa, sino que abarca diferentes esferas sociales. El concepto permite escapar de una visión esencialista sobre el periodismo, situándolo como una realidad socialmente construida a partir de la interacción simbólica entre los diferentes actores.

PALABRAS CLAVE: periodismo, mundo social, interaccionismo simbólico, transformación, identidad y prácticas.
This paper proposes to discuss the pertinence of the sociological concept of "social world" applied to journalism studies. Founded at symbolic interactionism tradition, this concept is generally used to study phenomenon which social recognize exists, but not necessarily in an institutionalized space. In this case, we understand that journalism comprehension must not be limited to newsmaking process, but is extensible to different social spheres. This concept allows escaping from an essentialist vision about journalism to define it as a social reality constructed by symbolic interactions between different actors.

KEYWORDS: journalism, social world, symbolic interactionism, changing, identity and practices.

1 Universidad de Brasilia. Brasil.

Correo electrónico: fabiohpereira_unb@yahoo.com.br 
En los últimos años, los estudios sobre periodismo en Brasil y en el mundo lusófono, en general, han experimentado grandes avances. La creación de la Asociación Brasileña de Investigadores en Periodismo (en portugués, SBPJor), de grupos y redes de investigación, ha contribuido al fortalecimiento institucional del campo. Al mismo tiempo, se observa un esfuerzo de sistematización de estudios y de la creación de un cuerpo teórico específico de esa área del conocimiento (Ver Pena, 2005; Sousa, 2000; Traquina, 1993, p. 167-176, 2001 y 2005).

Además de dar continuidad a este esfuerzo de sistematización y producción teórica, nos parece importante discutir un poco más sobre la necesidad de aplicar al periodismo teorías y estudios que puedan explicar mejor sus dinámicas de transformación y funcionamiento. Descuidar ese tipo de enfoque puede causar la falsa impresión de que existe una supuesta "naturaleza" de la práctica periodística. En cuanto constructo histórico, el periodismo está inmerso en la procesualidad del medio social. Así como su producción debe situarse como una práctica simbólica que construye socialmente la realidad, el propio "objeto periodismo" debe ser visto como integrante de esa realidad socialmente construida (Pereira, 2007a). Esa forma de ver la actividad que tienen sociólogos, antropólogos e historiadores también debe ser compartida por investigadores del propio campo de la comunicación. ${ }^{2}$

Algunas discusiones sobre ese enfoque ya fueron realizadas por el grupo internacional de la Réseau d'etudes sur le journalisme (REJ), compuesto por investigadores brasileños, franceses, canadienses y mexicanos. Como resultado del debate, se efectuaron dos publicaciones colectivas (Ringoot \& Utard, 2005; Augey, Demers \& Tétu, 2008). En ellas algunos autores contraponen la idea de la práctica periodística en cuanto "forma-

2 La dificultad, de hecho, está en articular esas dos instancias de construcción de la realidad. En ese sentido, Boyer \& Hannerz (2006, p. 8) afirman lo siguiente: "The ethnography of journalists offers more fine-grained insights, on the one hand, into the mediating practices of representation and circulation without which there would be no media, and, on the other, into the institutional and professional schemes and technical instrumentaria that wreathe, suffuse, and to some extent set conditions of possibility on the mediating labors of journalism." 
ción discursiva" (Foucault, 1969; Ringoot, et al., 2005), a otros conceptos pertinentes al análisis del objeto, como el de "campo periodístico” (Bourdieu, 1997, 2002; Champagne, 1993, 2004; Marchetti, 2002) y la noción de "paradigmas del periodismo" (Brin, Charon \& Bonville, 2004).

Definido en cuanto "formación discursiva" -término tomado por Ringoot, et al., (2005) de la semiología de Michel Foucault- el periodismo aparece como una práctica cuyo objeto, modos de enunciación, conceptos y estrategias no presentan una unicidad sociodiscursiva. Por el contrario, se encuentran de forma discontinua, "dispersos" en el interior de la formación. Desde el punto de vista de las formaciones discursivas, esa heterogeneidad debe ser aceptada como constitutiva del periodismo; a partir del análisis de sus reglas de dispersión es posible atribuir una identidad sociodiscursiva a esa práctica y distinguirla de un conjunto de saberes vecinos que se confunden y se entrecruzan:

La formación discursiva periodística se define, por tanto, como un centro de tensión entre orden y dispersión. Orden y dispersión de las informaciones, de las enunciaciones, de las estrategias. Esa noción de dispersión permite concebir la heterogeneidad del periodismo como constitutiva e intrínseca [...]. En esta apropiación conceptual, se trata de considerar cómo un discurso identificado e identificable se constituye por la acción de factores y de actores heterogéneos ${ }^{3}$ (ibidem, p. 42-43, traducción propia).

El concepto de campo, por su parte, tiende a situar el periodismo como un espacio social definido por leyes propias de funcionamiento, por relaciones de desigualdad y por dinámicas permanentes de disputa entre los agentes por las posiciones de dominación (Ringoot, et al. 2005). De acuerdo con esa perspectiva, es imposible analizar el espacio

3 "On définira donc la formation discursive journalistique comme un foyer de tension entre ordre et dispersion. Ordre et dispersion des informations, des énonciations, des stratégies. Cette notion de dispersion permet de penser l'hétérogénéité du journalisme comme constitutive et intrinsèque (...) Dans cette appropriation conceptuelle, il s'agit de considérer comment un discours identifié et identifiable est constitué sous l'action des facteurs et d'acteurs hétérogènes". 
periodístico sin situarlo en una red de dependencias con los campos político, económico e intelectual, cuyas lógicas de dominación determinan las modalidades de funcionamiento y de transformación de esa actividad. Ese mecanismo explica la diversidad de posturas y trayectorias observadas en el periodismo (habitus), sin que eso suponga necesariamente una pérdida de identidad o una constante subversión de la estructura de funcionamiento de ese campo.

Los paradigmas del periodismo, a su vez, hacen referencia a la constitución de un sistema de reglas coherentes sobre la práctica periodística, con alto grado de compatibilidad, que son utilizadas consensualmente por los participantes (Charron \& Bonville, 2004). Serían momentos en los que existiría una relativa homogeneidad de elementos discursivos de la práctica periodística, lo cual permitiría su tipificación. Al aplicar empíricamente ese concepto, los autores canadienses Jean Charron y Jean de Bonville definen cuatro tipos de periodismo históricamente adaptados a las circunstancias sociales, a saber: de transmisión, de opinión, de información y de comunicación. Para los autores citados, tales paradigmas se transforman a consecuencia de cambios en las estructuras sociales con las que la prensa se relaciona. Vistas de esa forma, las alteraciones del paradigma periodístico tendrían la misma amplitud que aquellas que afectan a las estructuras en las cuales está inmerso. En realidad, habría una congruencia entre el periodismo y las estructuras sociales, en la cual variables intermedias contaminarían prácticas vecinas situadas en un mismo contexto espacio-temporal. Así, existiría una serie de parámetros constitutivos del paradigma que van del texto periodístico a la estructura económica, pasando por la acción de los profesionales, las organizaciones mediáticas, las fuentes de información, las prácticas culturales y valores, elementos que interaccionan en un contexto de transformación.

Las perspectivas que acabamos de presentar permiten, por tanto, enriquecer el debate en torno al periodismo en cuanto práctica social y discursiva. Al partir de diferentes puntos de vista, tales estudios se proponen comprender las tensiones subyacentes a la actividad periodística construida desde diferentes dialécticas: las relaciones entre individuo y sociedad, entre estructura e historia, entre homogeneidad y heterogeneidad de esa actividad, entre el plano simbólico (de las "palabras") y el plano concreto de la práctica social (de las "cosas"). 
Dada la relevancia de este debate, proponemos aquí incorporar a las investigaciones sobre el periodismo otro concepto con la misma ambición y teórica metodológica de los estudios presentados anteriormente. Se trata de la noción de "mundo social". En este artículo será abordada la pertinencia del término y su aplicabilidad. Para apoyar nuestros argumentos, haremos uso de ejemplos extraídos del mundo cotidiano de los periodistas, de trabajos ajenos, de otras investigaciones individuales y de nuestra tesis de doctorado, ${ }^{4}$ Los periodistas-intelectuales en Brasil: identidades, prácticas y transformaciones en el mundo social, cuyos análisis se fundamentaron en la aplicación directa del concepto.

\section{EL INTERACCIONISMO SIMBÓLICO}

Y LOS PRESUPUESTOS TEÓRICOS

DEL CONCEPTO DE MUNDO SOCIAL

El concepto de mundo social se apoya en un enfoque sociológico del interaccionismo simbólico. Grosso modo, esta perspectiva centra su análisis en la manera en que las líneas de comportamiento son elaboradas por los actores, teniendo en cuenta los límites de la acción de su interlocutor. Esos actores son apenas personas; puede haber interacciones con objetos físicos, grupos sociales, instituciones, conceptos y abstracciones. Cada interacción se fundamenta en un proceso complejo en el cual el individuo busca orientar sus acciones a partir de la forma en que interpreta y anticipa la reacción del otro. Una vez que se produce esta reacción, el sujeto reevalúa su línea de conducta y la orienta a la interacción subsiguiente.

Convergiendo con las perspectivas constructivistas (Schutz, 1967; Heritage, 1991), el interaccionismo simbólico resalta el carácter contextual de la acción social, en la medida en que las motivaciones subyacentes deben ser situadas en el acto de la interacción, en la forma en que el individuo define e interpreta el objeto con el cual se relaciona. Para

4 Intentaremos aquí aplicar las condiciones expuestas por Anselm Strauss (1992) para la realización de un buen trabajo de investigación: sensibilidad teórica, calidad del material empírico y, también, experiencia personal del investigador. 
ese enfoque, toda interacción es simbólicamente mediada y presupone que el actor social se oriente de acuerdo con la representación que hace del otro. La construcción de ese otro remite a una serie de juicios sobre el interlocutor, algunos vinculados a experiencias individuales, otros a contextos colectivos, a una dimensión que podríamos calificar de estructural (Mead, 1934; Strauss, Schatzman, Bucher, Ehrlich \& Sabshin, 1964; Strauss, 1992).

El interaccionismo simbólico busca, por lo tanto, incorporar dos dialécticas fundamentales a la comprensión de la sociedad. Primero, la idea de que toda interacción es un proceso de acción sobre el otro (individuo, grupo, comunidad), en el plano simbólico (de las palabras) y también en el plano concreto de la vida social (de las cosas). Segundo, que dicha relación se articula en las dimensiones estructural/sociológica e individual/psicológica.

La especificidad de esa perspectiva está precisamente en el hecho de que la interacción simbólica debe ser entendida como un locus privilegiado de análisis de los fenómenos sociales. Los interlocutores implicados en el proceso interactivo orientan, confrontan, confirman o modifican sus visiones de mundo y prácticas teniendo en cuenta la relación con el otro. En ese sentido, la interacción adquiere un carácter evolutivo y transformador (Strauss, 1992) que construye la identidad y la conducta individual, al mismo tiempo que funciona como instancia de construcción de la realidad social.

Partiendo de esos presupuestos, el concepto de "mundo social" constituye lo que Merton (1970) llama "teoría social de medio alcance" (middle range theory), un modelo analítico capaz de aproximar las abstracciones de carácter teórico expuestas por el interaccionismo simbólico a los eventos observados en la vida cotidiana de las sociedades. Además, traduce y amplía la idea de interacción en un conjunto de conceptos y procedimientos que abarcan desde la identidad y las prácticas individuales hasta los cambios más amplios de orden estructural. En ese sentido, defendemos su aplicabilidad en los estudios sobre periodismo.

\section{EL MUNDO DE LOS PERIODISTAS}

Un mundo social consiste en una red de personas implicadas en la realización de una actividad cooperativa (Becker, 1982; Gilmore, 1990; 
Strauss, 1992). Estas coordinan las prácticas una vez que tienen como base un cuerpo de entendimientos, intereses y artefactos necesarios para la realización de un acto social mayor. Los mundos son diferentes de las instituciones y de las organizaciones, ya que sus dinámicas de funcionamiento no están necesariamente fundamentadas en relaciones de poder, autoridad o dominación (Gilmore, 1990). Además, la participación de los individuos no depende de una pertenencia institucional; está asociada únicamente a las formas convencionales de actuar en la realización de esa actividad. Por ello:

Pertenecer a todos esos mundos sociales implica compromisos variados de orden general que van más allá de los compromisos específicos y son fácilmente perceptibles en las oficinas, instituciones, organizaciones, camarillas y segmentos especializados de la sociedad en relación con el mundo social 5 (Strauss, 1992, p. 173, traducción del autor).

Con excepción del libro $O$ mundo dos jornalistas, de Isabel Travancas (1992), se constata una falta de aplicación de ese concepto en los estudios de comunicación en Brasil. Por ello, para poder trabajar con dicho concepto, recurriremos a algunas definiciones adaptadas de la obra de Howard S. Becker (1982), Art Worlds (Mundos del arte), y del mencionado libro de Travancas.

\section{Redes de cooperación y la extensión de un mundo social}

Un mundo social está formado por una "red de cooperación" (net of cooperation) cuyos actores participan, en diferentes grados, en la actividad final. Esa red no se limita a lo que Becker llama "núcleo" (core) de un mundo social, es decir, a las actividades convencionalmente aso-

5 "Appartenir à tous ces mondes sociaux implique des engagements variés d'ordre général que dépassent les engagements plus spécifiques et facilement perceptibles pour les bureaux, les institutions, organisations, cliques et spécialités en relation avec le monde social." Generalmente, el concepto de "mundo social" es utilizado para analizar fenómenos, imprecisos y flexibles, cuyo reconocimiento social existe sin necesidad de que estén ubicados en un espacio institucionalizado o en una organización social (Gilmore, 1990). 
ciadas a una práctica social, sino que se extiende por toda la sociedad, en interacción con diversos espacios, dominios y actores.

De acuerdo con el sentido común, el periodismo está asociado a un conjunto de actividades que componen su núcleo, generalmente conectadas a la producción informativa (apuración, redacción y edición), realizada por periodistas, a partir del espacio de las redacciones (Bahia, 1990; Kunczik, 1997; Ribeiro, 1994). Esa definición está suficientemente arraigada en las sociedades y orienta una parte significativa de las investigaciones etnometodológicas sobre las rutinas de producción de los periodistas, realizadas a partir de los años setenta (Traquina, 1993, 2001).

Sin embargo, las redes de cooperación que componen el mundo de los periodistas van mucho más allá de esas prácticas. Por un lado, los productos periodísticos impregnan otros teatros de la experiencia social con objetivos diferentes del papel informativo atribuido al periodismo (Boyer, et al., 2006). Por otro, su producción dentro de las redacciones depende de un número expresivo de actores sociales no periodistas, como office boys, secretarias, técnicos en informática, guardias de seguridad, gerentes y directores de empresas de comunicación (Travancas, 1992). Incluye también a colaboradores externos, como las fuentes, el público, los articulistas, los cronistas, los asesores de prensa, los anunciantes, los publicitarios, los dueños de quioscos, los grafistas, los productores de papel y tinta, etc. Sin ellos, un periódico no podría salir o no saldría de la manera como normalmente es concebido.

El concepto de red de cooperación, su variable extensión y las relaciones que establece en la producción de una mayor acción social que caracteriza el mundo de los periodistas, es fundamental para que podamos comprender aspectos del periodismo que escapan a los enfoques de carácter esencialista y funcionalista. Los cuestionamientos sobre las rutinas productivas de un periódico no pueden ignorar las interacciones establecidas con las fuentes (Hall, Chritcher, Jefferson, Clarke \& Roberts, 1993; Schlensiger, 1992) y con el público (Gadini, 2007; Ruellan, 2006). Asimismo, los despachos enviados por las agencias de noticias y por los sectores de comunicación institucional ocupan un lugar cada vez mayor en la composición del noticiario. De hecho, hoy día en Brasil una huelga de los grafistas o un corte en la producción de papel tendría más probabilidades de 
perturbar, o incluso de impedir, la publicación de un periódico impreso que una paralización de los periodistas, los cuales, por definición, ocupan un lugar central en la producción del noticiario.

En el campo de la identidad, dichas consideraciones ayudan a trabajar mejor los conflictos sobre quién debe ejercer las actividades consideradas esenciales al mundo social. Si el periodismo no depende únicamente de las prácticas de reportaje, figuras externas a la redacción, como los asesores de prensa y los periodistas-intelectuales, pueden, en algunos casos, reivindicar la identidad de periodistas y su inserción como integrantes de ese espacio que podría ser redefinido.

En el caso de la comunicación institucional, todo el debate sobre la atribución del estatuto de periodista a los profesionales que trabajan en asesorías de prensa, gira en torno a lo que debe ser definido como núcleo o "naturaleza" de la práctica periodística. De acuerdo con las conclusiones a las que llegamos en trabajos anteriores (Pereira, 2006; 2007b), los argumentos contrarios a la inclusión de los asesores en el núcleo del periodismo se refieren, fundamentalmente, a la idea de que solo las empresas de comunicación garantizan la credibilidad y legitimidad necesarias para que un trabajo pueda ser considerado realmente periodístico. Los defensores, por otro lado, sitúan la definición de ese núcleo en otro nivel, en el cual el proceso de difusión de informaciones de interés público no depende del tipo de institución involucrada en esa actividad. Este sería el caso de los asesores de prensa.

Se observa, en este caso, una situación problemática que la reflexión meramente esencialista (" $¿ C u a ́ l$ es la verdadera naturaleza del periodismo?") es incapaz de aclarar. De ahí que sea necesario no limitar el análisis a un discurso cerrado sobre la esencia de esta práctica y a las visiones formuladas desde dentro por el grupo profesional, sino trabajar también con las prácticas de otros integrantes del mundo social. Sant'Anna (2005) y Moura, Adghirni, Pereira, Sant'Anna y Silva (2008) muestran cómo las acciones desarrolladas por las entidades sindicales (que definen al periodista por la posesión del registro profesional, obtenido con una formación universitaria en periodismo, aunque trabaje como asesor de prensa), por las instituciones no-mediáticas, públicas y privadas (que prefieren contratar a periodistas profesionales para actuar como comunicadores en sus servicios de prensa) y por el propio público (que puede eventualmente 
reconocer y legitimar prácticas y profesionales de comunicación institucional como pertenecientes al mundo de los periodistas) explican mejor el proceso de expansión de las fronteras profesionales y de redefinición de la esencia, del núcleo, de esa actividad en Brasil (Ruellan, 1993).

De la misma manera, al analizar a los periodistas-intelectuales brasileños -profesionales que se caracterizan por poseer relaciones de doble pertenencia o identidad, y que transitan en espacios de convivencia entre la prensa, el medio político y el intelectual- exploramos toda la extensión de la red de colaboradores de ese mundo social (Pereira, 2008). Observamos, en este caso, que además de las interacciones realizadas con actores situados muy próximos al núcleo del periodismo -los pares periodistas, las fuentes y el público- los periodistas-intelectuales deben parte de su estatus a las relaciones con diferentes grupos sociales, presentes a lo largo de sus trayectorias socioprofesionales. Destacamos, en este caso, la acción de los intelectuales, el Estado, los editores y críticos literarios, los partidos políticos, los sindicatos y los movimientos sociales, y los alumnos de cursos de periodismo, entre otros, como corresponsables de la atribución de la identidad del grupo. Nuestras conclusiones apuntan también a la reputación, que es adquirida dentro de una especie de mosaico de interacciones. Ese carácter fragmentario ofrece una descripción bastante adecuada del mundo social, de su variable extensión y de la imprecisión de las fronteras que rigen la actividad periodística.

\section{Periodismo y convenciones}

Todo mundo social está marcado por un conjunto de convenciones (conventions) que deciden los términos de la cooperación, hacen que las decisiones sean más simples y proporcionan la base para que los participantes puedan actuar juntos y de manera eficiente en la producción de un trabajo. ${ }^{6}$ Siguiendo ese punto de vista, la periodicidad de un medio, las rutinas de una redacción (pauta, investigación, redacción, edición, diagramación) y las técnicas periodísticas (lead o entradilla y pirámide

6 En ese sentido, las convenciones corresponden, en una dimensión más amplia, al sistema normativo que delimita ciertas características de una interacción, permitiendo que los actores anticipen las reacciones del otro y orienten así sus acciones. 
invertida) se constituyen en convenciones corrientes en el mundo de los periodistas. Desde el punto de vista convencional, el proceso de producción de noticias se observa como una dinámica interactiva, "donde diversos agentes sociales ejercen un papel activo en el proceso de negociación constante" y cuya necesidad de prever la cobertura de los hechos se materializa en un conjunto de rutinas productivas (Traquina, 2001, p. 64).

Las convenciones varían conforme al nivel de formalización. Pueden ser sistematizadas en el mundo de los periodistas por medio de códigos deontológicos, leyes, manuales de redacción y un cuerpo de conceptos y teorías, presente en libros y enseñado en las universidades. O pueden existir de manera menos formal y ser compartidas únicamente por los que participan en una determinada actividad directamente relacionada con el oficio. Parte de esas convenciones es socializada a todos los integrantes del mundo social para coordinar su participación en la red de cooperación. Un asesor de prensa necesita estar atento a las rutinas y a la línea editorial de un periódico. El público, generalmente, conoce los formatos narrativos canónicos del periodismo. ${ }^{7}$ Una empresa de publicidad debe conocer los plazos (deadlines) y la línea editorial del medio en el que quiere anunciar.

Ciertas convenciones están tan estrechamente relacionadas con un mundo social específico, que son experimentadas como si fuesen parte de la cultura, de la estructura social. La periodicidad de los periódicos es un ejemplo típico de ese fenómeno. Otras son lo suficientemente estables en el mundo social como para ser utilizadas en la formación de nuevos miembros. Es el caso de las técnicas de redacción enseñadas en los cursos de periodismo. Algunas poseen carácter normativo o se proponen defender al grupo social. La noción de objetividad en el periodismo, la regla de oír a las dos partes y los códigos deontológicos forman parte de ese tipo de convenciones.

Aun cuando están arraigadas en el mundo social, las convenciones no son inmutables. Como parte integrante de un orden negociado, dan margen a formas distintas de interpretación y cambio. "Las convenciones representan la adaptación continua de las partes cooperantes al

7 Lo que los semiólogos llaman "horizontes de lectura" (Ringoot, 2006). 
cambio de las condiciones en las cuales operan; cuando las condiciones cambian, las convenciones también cambian"8 (Becker, 1982, p. 59, traducción del autor).

De hecho, las convenciones difícilmente consiguen cubrir todas las situaciones vividas en el ámbito de un mundo social. Eso da margen para la introducción de innovaciones destinadas a resolver contextos específicos. Algunas de ellas pueden ser incorporadas al mundo social, siempre que sean aceptadas por los demás miembros. Fue lo que sucedió en la década de 1950 en Brasil, con la introducción de técnicas como el lead o entradilla, la pirámide invertida y la noción de objetividad. Otras convenciones pueden quedar restringidas a un contexto o a un grupo de colaboradores (como en el caso del nuevo periodismo norteamericano) o pueden incluso desaparecer (como el antiguo estilo de la "nariz de cera", introducciones literarias o filosóficas utilizadas en textos periodísticos brasileños hasta mediados del siglo XX).

La opción de innovar o seguir utilizando las convenciones en el mundo social remite a la forma en que un miembro concilia sus intereses (de experimentación o solución de un problema específico) y cómo esos cambios son aceptados y compartidos por los demás participantes. Siempre es posible hacer las cosas de un modo diferente, aunque se pague un precio por ello: más esfuerzo, menos circulación, pérdida del empleo, etc. Por ese motivo, el mundo social puede ser visto como una combinación de aspectos convencionales e innovadores (Becker, 1982).

\section{Opciones y reputación}

La relación entre el individuo y el mundo social es cotidianamente vivenciada en las opciones (choices) realizadas por los participantes. Las opciones son, en realidad, interacciones que un miembro realiza con los demás actores implicados en las actividades de cooperación del mundo social o que cuenta con ellos. Las opciones pueden o no ser verbalizadas y siempre consideran la existencia de un interlocutor, aunque no sea necesariamente una persona. A partir de ellas, el sistema convencional

8 "Conventions represent the continuing adjustment of the cooperating parties to the changing conditions in which they practice, as conditions changes, they change." 
es puesto en práctica, puede ser aceptado o no y llevar, o no, las innovaciones al mundo social. El mayor acto social que caracteriza un mundo es, en realidad, el resultado de esas permanentes opciones. Vehicular una noticia a través de un periódico puede ser definido de esa forma. Esto implica, por ejemplo, la elección de la pauta, las fuentes, las preguntas hechas al entrevistado, el encuadramiento adoptado, la mejor forma de redactar, editar y diagramar, el horario de cierre, el papel y el tipo de impresión adoptados, la forma de distribución, la decisión de comprar el periódico, de leer determinada noticia, de cómo interpretarla, etc.

Las opciones subyacentes a la producción periodística no son necesariamente racionales o coherentes -aunque una mirada rápida pueda dar la impresión de que están guiadas por el mantenimiento de la funcionalidad del sistema. Sin embargo, cuando integramos este concepto a los análisis de rutinas productivas se evidencia cómo, incluso en situaciones cotidianas cubiertas por el sistema de convenciones, el mundo social siempre implica negociación entre diferentes actores (Boyer, et al., 2006). En la medida en que incluso las situaciones más convencionales de la producción periodística dan margen para la decisión individual o colectiva de las personas implicadas, es posible comprender mejor la tensión entre la estabilidad sociocognitiva de esa actividad y los diferentes niveles de innovación y cambio que afectan a la actividad periodística (Charron, et al., 2004).

El concepto de opciones también puede ser aplicado en los análisis microsociológicos sobre el trayecto de un grupo socioprofesional. En ese caso, permiten explicar cómo la conquista de un estatus o de una posición de mundo social no es fruto ni del acaso ni de un orden lógico y unidireccional -aunque algunas opciones puedan ser previstas en el ámbito de las carreras profesionales (Strauss, 1992)-, sino de las dinámicas de interacción simbólica. En la investigación sobre los periodistas-intelectuales observamos cómo un grupo aparentemente análogo reflejaba una diversidad de opciones -comprometerse políticamente, publicar un libro, ingresar en una universidad, etc.-, dispersas en el transcurso de sus historias de vida. Esas opciones, a la vez que explican la reputación alcanzada por esos actores, remiten a la relación de ese estatus con el contexto estructural, sin que se conviertan necesariamente en caminos naturales u obligatorios en la construcción de esas carreras. A partir de 
este punto, nuestro trabajo se aproxima a un concepto similar utilizado por la sociología burdiesana, la noción de habitus, aunque sin aferrarnos a las estrictas relaciones de dominación establecidas por Bourdieu (2002) entre esas trayectorias y las lógicas de funcionamiento del campo.

Por lo tanto, a partir de las opciones se construye la reputación de los individuos en el mundo social. El concepto de reputación está relacionado con el procedimiento de atribución de identidad por parte del otro durante el proceso interaccional. No obstante, la reputación no se limita al cara a cara de la interacción. Toda reputación es, de alguna manera, compartida por los demás miembros del mundo social. Asimismo, puede ser atribuida no sólo a una persona, sino a un grupo, a una institución o a una comunidad. Es el caso de la reputación atribuida a los jazzistas de New Orleans (Becker, 1982) o a los periodistas-escritores de la revista New Yorker (Costa, 2005).

La reputación de un actor social depende no sólo de las opciones, conscientes o no, que realiza en su trayectoria dentro del mundo social, sino también de la base convencional vigente en un determinado momento. En realidad, existe una relación entre lo que es aceptable en términos de mundo social (negociado con los demás miembros), la reputación de los actores implicados y la decisión de mantener un determinado conjunto de convenciones o romper con ellas.

Las reputaciones no son consideradas de la misma manera por todos los miembros de una actividad. Ciertas prácticas que antiguamente garantizaban la reputación de un periodista -el compromiso político o literario, por ejemplo- pasaron a ser mal vistas a partir de 1950. Asimismo, un periodista que tiene una mirada crítica sobre la profesión puede adquirir mala reputación entre los compañeros y, por otro lado, poseer legitimidad en el medio académico.

\section{Transformaciones en el mundo de los periodistas}

Además de explicar las dinámicas de funcionamiento del periodismo, el concepto de mundo social ofrece líneas de análisis para las grandes transformaciones que se han producido en este ámbito. En sus trabajos sobre el medio artístico, Becker (1982) habla de procesos que producen cambios evolutivos o revolucionarios de los mundos sociales y también de fenómenos más localizados de segmentación que afectan a un 
grupo restricto de actores y prácticas. La misma lógica puede aplicarse al periodismo. Para analizar sus transformaciones es necesario establecer el nivel de alteración de las actividades de cooperación y del lenguaje convencional que pueden desencadenar cambios graduales o revolucionarios (Becker, 1982). En un mundo social, estos cambios pueden tener su origen, por ejemplo, en la difusión de nuevas tecnologías y conceptos o en la introducción de una nueva audiencia. Como consecuencia, se producen cambios en esa actividad, desde un punto de vista ideológico y también organizacional.

Esos presupuestos permiten introducir operadores para analizar cómo y por qué el periodismo se (re)configura en un determinado periodo histórico. La aparición del "webperiodismo", por ejemplo, es el resultado directo de una nueva base convencional introducida a partir de una innovación tecnológica (Internet y sus características específicas), y también de alteraciones en la red de colaboradores del mundo social. Las consecuencias de ello son la construcción de un nuevo perfil de periodista profesional para trabajar en los periódicos web (Adghirni, 2002; Pereira, 2004), el surgimiento de nuevos miembros en la red de cooperación -programadores, webmasters y webdesigners, de empresas creadas para explotar el nuevo filón que supone Internet, etc.-, y también una alteración en el modo convencional de colaboración de los actores en la producción periodística. En una investigación realizada en 2003 verificamos una intensificación del aprovechamiento por parte de los nuevos sitios de Internet del material enviado por las agencias para la producción de noticias (Pereira, 2004). En ese caso, Internet alteró el proceso de gatekeeping y el estatus de los actores externos implicados en la producción periodística.

El concepto de mundo social fue aplicado en el análisis de las transformaciones del periodismo y sus relaciones con el espacio intelectual. En ese caso tratamos de investigar y cuestionar el proceso del autonomización y profesionalización del periodista frente a los demás grupos intelectuales a partir de la década de 1950. Esos cambios fueron desencadenados por la introducción de nuevas convenciones en las redacciones, hecho que ocurrió paralelamente a una depuración de los antiguos literatos y un ataque ideológico al viejo orden (los manuales de redacción y la adopción de ideas de objetividad periodística son ejemplos de ello). No obstante, el proceso sólo pudo ser completado a partir de 
una reorganización más profunda de las redes de cooperación de todo el medio político y cultural brasileño, lo que permitió que los antiguos intelectuales se reinsertaran en el mercado profesional -como escritores cineastas, profesores, etc.-, pasando así a intervenir en el periodismo bajo nuevas condiciones.

Ese proceso, en realidad, ya ha sido analizado por investigadores del área. Lo interesante, en este caso, es evaluarlo a partir de una dialéctica que tome en cuenta el modo en que fue vivido por los actores (los periodistas-intelectuales) y cómo esos cambios se reflejan en un modelo explicativo sobre el espacio periodístico (el concepto de "mundo social").

Si tomamos como presupuesto que un mundo social posee una expansión mucho más variable de lo que se observa en las actividades que componen su núcleo, debemos extender también el análisis sobre sus transformaciones a otros actores y espacios sociales que interaccionan con él. De hecho, nuestro estudio sobre las transformaciones en el periodismo brasileño es indisociable de un análisis de los medios político y cultural. Revela cómo la profesionalización del militantismo o la introducción de un sistema de titulación universitaria en el país están directamente relacionados con el surgimiento de un nuevo perfil de periodista. En consonancia con los conceptos de "campo" (Bourdieu, 1997; 2002), "frontera" (Ruellan, 1993) y "formación discursiva" (Ringoot, et al., 2005), lo que se plantea aquí es que la comprensión del espacio periodístico no se limita al discurso emitido por dentro, sino que se realiza siempre desde un punto de vista interdiscursivo, interaccional e históricamente situado. En ese sentido, el concepto de mundo social se une a los demás como un modelo analítico que abre grandes posibilidades en términos de alcance y aplicación en futuras investigaciones sobre el periodismo.

\section{EL CONCEPTO DE MUNDO DE LOS PERIODISTAS: PERSPECTIVAS METODOLÓGICAS}

Los análisis que haremos a continuación sobre la aplicación y operacionalización del concepto de mundo social en estudios empíricos en las áreas de comunicación y periodismo no se fundamentan en una prescripción metodológica cerrada, al estilo, por ejemplo, de un manual de méto- 
dos y técnicas de investigación. Aunque el propio Howard Becker (1997; 2008) se haya destacado en el medio académico por la publicación de trabajos en el área de metodología de investigación, su definición sobre el mundo de las artes se basa más en la presentación de las conclusiones teóricas y empíricas derivadas de ese concepto que en la creación de un conjunto de procedimientos metodológicos específicos para analizar un mundo social.

No obstante, si partimos de la lectura de las investigaciones de campo de Becker (1982), Strauss (et al., 1964), Travancas (1992) y de nuestras propias estrategias metodológicas (Pereira, 2006, 2008), es posible delimitar algunas líneas de acción que pueden ser útiles en la realización de investigaciones en el área del periodismo a partir del concepto de "mundo social". De modo general, tales lecturas indican las posibilidades de utilización y cruce de una gama variada de metodologías y de técnicas ligadas a la tradición cualitativa de las investigaciones en ciencias sociales. Esa vinculación con los métodos cualitativos traduce, en realidad, la propia visión epistemológica del interaccionismo simbólico y de la Escuela de Chicago. Esta perspectiva implica la elaboración de proyectos metodológicos que procuren conciliar un trabajo exhaustivo de investigación de campo, una estrecha vinculación entre el investigador, las comunidades y los sujetos investigados, y flexibilidad en los procedimientos de investigación adoptados (Becker, 1997).

Howard Becker destaca tres métodos principales utilizados en investigaciones de campo etnográficas ligadas al interaccionismo: la observación participante, la recogida de testimonios por medio de entrevistas informales o estructuradas y el análisis de registros, como documentos, cartas, etc. A continuación analizaremos brevemente estos métodos y su aplicación en los estudios sobre periodismo.

Con la observación participante se busca conocer detalladamente lo cotidiano de una determinada comunidad con el objetivo de comprender los modos convencionales de actuación en el mundo social (Becker, 1982), así como las articulaciones entre identidades, carreras e ideologías profesionales (Strauss, et al., 1964). Esto es lo que han hecho generalmente los estudios de sociología de los emisores y newsmaking. Tales investigaciones tienden a centrarse en la observación del día a día de las redacciones y consideran a la comunidad de periodistas como 
una especie de tribu, depositaria de normas de comportamiento y valores sociales y profesionales característicos.

La existencia de un modo de ver (la estética periodística), de un modo de hablar (la jerga periodística) y de un modo de actuar (la epistemología periodística) establece un vínculo bastante fuerte entre los miembros de la tribu periodística (Traquina, 2001, p. 122).

La relación entre observación participante y mundo social supone definir el escenario de análisis como todo el locus de vivencia de los periodistas y de su red de colaboradores. Es lo que hace Isabel Travancas (1992) al extender el estudio sobre la identidad profesional de los periodistas más allá del espacio de la redacción, relacionando tal identidad, también, con lo que sucede en la calle y en los espacios privados. De la misma forma, se puede comprender el funcionamiento del mundo de los periodistas sin analizar necesariamente su vida cotidiana. Es posible, por ejemplo, centrar una investigación en este campo a partir de los estudios sobre lo cotidiano de las fuentes de información (Hall, et al., 1993), del público o de actores sociales que desempeñan actividades no periodísticas o paraperiodísticas. En esa línea se sitúan los estudios de las investigadoras Fillion y Le Cam (2007) sobre los blogs periodísticos y los documentales, y el análisis de Olivier Tredan (2009) sobre las transformaciones del mundo de los periodistas, observadas a partir de los blogs de adolescentes en Francia.

La recolección de testimonios y la reconstrucción de las historias de vida de ciertos actores o grupos sociales es otro método importante, sobre todo para el análisis de la dimensión histórica o longitudinal de un fenómeno. Se trata de articular cuestiones ligadas a las identidades o prácticas de los sujetos investigados con un conjunto de macrotransformaciones sociales. Como explican McCall y Wittner (1990), al relatar sus historias, las personas, en realidad, buscan compartir con el investigador las soluciones colectivas y los sentidos atribuidos a sus experiencias sociales. En ese caso, es necesaria una compleja articulación entre las acciones individuales presentadas, las acciones de los demás miembros de la red de cooperación, las reglas institucionales y las convenciones del mundo social y las estructuras históricas. 
Ese tipo de análisis requiere, por tanto, un tratamiento diferente de los testimonios recogidos, que no pueden ser definidos como simples datos de investigación sino como situaciones de interacción en las que el entrevistado busca obtener significados de su experiencia, teniendo en cuenta a su interlocutor:

Si alguien observa las entrevistas no como registros sino como escenarios interactivos para la construcción de significados, el papel del entrevistador deja de ser totalmente pasivo y neutro. Con independencia de que estén planeadas o no -y de que nos gusten o no-, las narrativas del entrevistado están influenciadas por la interacción social que se desarrolla en la entrevista y por la actitud del entrevistado en relación con la interpretación de la narrativa ${ }^{9}$ (Järvinen, 2003, p. 225-226, traducción propia).

Aplicamos ese método, con relativo éxito, en el estudio sobre la identidad y las prácticas de los periodistas-intelectuales. Nuestro objetivo era relacionar el contexto de la entrevista con el estatus atribuido por los entrevistados al investigador. En un segundo momento, buscamos inferir cómo ese proceso de atribución de sentido podría ser extendido a los demás participantes del mundo social que interaccionaron en diferentes situaciones con los periodistas-intelectuales entrevistados y con el propio escenario de transformaciones del espacio político y cultural en Brasil. En este caso, consideramos que no podíamos limitarnos a analizar las explicaciones de los entrevistados, sino que debíamos comprender también el contexto en que esas explicaciones eran articuladas. Se trataba de centrarse en el proceso de construcción de esas identidades, en lugar de establecer simplemente un concepto definitivo sobre el grupo (Becker, 2008).

Finalmente, la investigación documental utiliza estrategias metodológicas semejantes a la recolección de testimonios o a la realización de ob-

9 "If one regards interviews not as recording but as interactive sites for meaning making the interviewer's role cannot be totally passive and neutral. Whether planned or not - and whether we like it or not - the interviewer's narratives are influenced by the social interaction going on in the interview, and by the interviewer's attitude towards and interpretation of the narrative". 
servaciones in situ. La diferencia está en la decisión de trabajar con otros tipos de fuentes de datos, según los objetivos, las limitaciones y las características de la investigación en cuestión. La lectura de correspondencia, por ejemplo, permitió a Becker (1982) analizar en Art Worlds el papel desempeñado por editores y amigos en el proceso de creación artística, algo que tradicionalmente era considerado por la teoría estética como resultado exclusivo del talento individual del autor. En su estudio sobre los blogs de adolescentes, Olivier Tredan (2009) también utiliza el concepto de mundo social para analizar los comentarios e hipervínculos utilizados por los "blogueros" para administrar sus universos de sociabilidad. En la construcción y exposición de sus identidades individuales por medio de los blogs, los adolescentes se apropian de contenidos "externos" al universo de la "blogosfera", como producciones mediáticas y periodísticas.

\section{CONSIDERACIONES FINALES}

En este breve texto hemos analizado la aplicación del concepto de "mundo social" en los estudios de las dinámicas de funcionamiento y transformación del periodismo. Ciertamente, se trata de un primer esfuerzo de teorización, de una exposición todavía limitada sobre los modos de utilización de este enfoque. No obstante, nos parece bastante apropiado para analizar resultados empíricos, sobre todo en investigaciones etnográficas sobre el periodismo, dentro de un esfuerzo de comprensión más amplia de ese fenómeno social.

\section{Bibliografía}

Adghirni, Z. L. (2002). Jornalismo online e identidade profissional do jornalista. En L. G. Motta (Ed.), Imprensa e Poder (pp. 151-166). Brasília: Editora UnB.

Augey, D., Demers, D., Tétu, J.-F. (Orgs.) (2008). Figures du journalisme. Brésil, Bretagne, France, La Réunion, Mexique, Québec. Québec: PUL.

Bahia, J. (1990). Jornal, História e Técnica - as técnicas do jornalismo ( $4^{\mathrm{a}}$ ed.). São Paulo: Ática.

Becker, H. S. (1997). Métodos de pesquisa em Ciências Sociais ( $\left.3^{\mathrm{a}} \mathrm{ed}.\right)$. São Paulo: Hucitec. 
Becker, H. S. (1982). Art worlds. Berkeley \& Los Angeles: University of California Press.

Becker, H. S. (2008). Os segredos e truques de pesquisa. Rio de Janeiro: Jorge Zahar Editores.

Bourdieu, P. (1997). Sobre a Televisão. Río de Janeiro: Jorge Zahar Editores.

Bourdieu, P. (2002). Quéstions de sociologie. París: Les Editions de Minuit.

Boyer, D. \& Hannerz, U. (2006). Introduction: Worlds of Journalism. Ethnography, 1 (7), 5-17.

Brin, C., Bonville, J. \& Charron, J. (Eds.) (2004). Nature et transformation du journalisme. Théories et recherches empiriques. Québec: Les Presses de L'Université Laval.

Champagne, P. (1993). La vision médiatique. En P. Bourdieu (Ed.), La misère du monde (pp. 95-123). París: Éditions du Seuil.

Champagne, P. (2004). Sur la télévision. En P. Champagne, R. Chartier (Eds.), Pierre Bourdieu et les médias. Rencontres Ina/Sorbonne (pp. 43-51). París: L'Harmattan.

Charron, J. \& Bonville, J. (2004). Typologie historique des pratiques journalistiques. En C. Brin, J. Charron \& J. Bonville (Eds.), Nature et transformation du journalisme. Théories et recherches empiriques (pp. 141-217). Québec: Les Presses de L'Université Laval.

Costa, C. (2005). Pena de aluguel: escritores-jornalistas no Brasil: 1904-2004. São Paulo: Cia das Letras.

Fillion, N. \& Le Cam, F. (2007). Documentários e weblogs: uma extensão da identidade editorial jornalística? Comunicação \& Espaço Público, 1 e 2, Ano X, 50-68.

Foucault, M. (1969). L’Archéologie du savoir. París: Gallimard.

Gadini, S. L. (2007). Em busca de uma teoria construcionista do jornalismo contemporâneo: a notícia entre uma forma singular de conhecimento e um mecanismo de construção social da realidade. Revista Famecos, 79-88.

Gilmore, S. (1990). Art worlds: developing the interactionist approach to social organization. En H. S. Becker \& M. M. McCall (Eds.), Symbolic interaction and cultural studies (pp. 148-178). Chicago \& Londres: The University of Chicago Press. 
Hall, S., Chritcher, C., Clarke, J., Jefferson, T. \& Roberts, B. (1993). A produção social das notícias: o mugging nos media. En N. Traquina (Ed.), Jornalismo: questões, estórias e “estórias” (pp. 224-248). Lisboa: Vega.

Heritage, J. C. (1991). L'Ethnomethodologie: une approche procédural de l'action et de la communication, Réseaux, 50, 89-123.

Järvinen, M. (2003). Negotiating Strangerhood: interviews with homeless immigrants in Copenhagen, Acta Sociologica, 3 (46), 215230.

Kunczik, M. (1997). Conceitos de jornalismo: Norte e Sul. São Paulo: EDUSP.

McCall, M. M. \& Wittner, J. (1990). The good news about life history. En H. S. Becker \& M. M. McCall (Eds.), Symbolic interaction and cultural studies (pp. 46-89). Chicago \& Londres: The University of Chicago Press.

Marchetti, D. (2002). Les sous-champs spécialisés du journalisme, Réseaux, 111, 21-56.

Mead, G. H. (1934). Mind, Self \& Society: From the Standpoint of a Social Behaviorist. Chicago: University of Chicago Press.

Merton, R. (1970). Sobre as teorias sociológicas de médio alcance. En R. Merton (Ed.), Sociologia: Teoria e Estrutura (pp. 51-79). São Paulo: Mestre Jou.

Moura, D. O., Adghirni, Z. L., Pereira, F. H., Sant'Anna, F. C. C. M. \& Silva, L. M. (2008). Journaliste un jour, journaliste toujours. En D. Augey, F. Demers \& J-F. Têtu (Eds.), Figures du Journalisme - Brésil, Bretagne, France, La Réunion, Mexique, Québec (pp. 131-145). Québec: Les Presses de L’Université Laval.

Pena, F. (2005). Teoria do Jornalismo. São Paulo: Editora Contexto.

Pereira, F. H. (2004). A produção jornalística na internet e a construção da identidade profissional do webjornalista. Anais do V Congreso Iberoamericano de Periodismo en Internet.

Pereira, F. H. (2006). Journalistes et attachés de presse à la Présidence de la République au Brésil: intérêts cachés et discours de mythification sur la fonction de la presse. Paper presentado durante la Journée d'étude "Journalisme et démocratie: un contre-pouvoir imaginaire? 
Pereira, F. H. (2007a). Jornalismo e Construtivismo: a atividade como realidade socialmente construída, Revista PJ:Br, V. 5. São Paulo. Recuperado el 05/01/2008, de http://www.eca.usp.br/pjbr/arquivos/ artigos9_e.htm

Pereira, F. H. (2007b). Jornalistas e assessores: limites e perspectivas para um debate sobre as identidades profissionais. En B. Lopes Filho (Ed.). Gestão em comunicação empresarial-Teoria e técnica (pp. 72-79). Juiz de Fora: Editora UFJF/Produtora de Multimeios da UFJF.

Pereira, F. H. (2008). Os jornalistas-intelectuais no Brasil: identidades, práticas e transformações no mundo social. Tese de doctorado inédita. Programa de Postgrado en Comunicación, Universidade de Brasília.

Ribeiro, J. C. (1994). Sempre Alerta: condições e contradições do trabalho jornalístico. São Paulo: Brasiliense.

Ringoot, R. \& Utard, J.-M. (2005). Genres journalistiques et “dispersion” du journalisme. En R. E. Ringoot \& J.-M. Utard (Eds.), Le journalisme en invention. Nouvelles pratiques, nouveaux acteurs (pp. 21-47). Rennes: Presses Universitaires de Rennes.

Ringoot, R. (2006). Por que e como analisar o discurso no contexto dos estudos sobre jornalismo? Comunicação e Espaço Público, 1 e 2, ano IX 133-139.

Ruellan, D. (1993). Le Professionnalisme du Flou. Identité et savoirfaire des journalistes français. Grenoble: Presses Universitaires de Grenoble.

Ruellan, D. (2006). A pesquisa em jornalismo e o interesse público: pensar o corte e a costura. Anais do IV Encontro Nacional de Pesquisadores de Jornalismo.

Sant'Anna, F. C. C. M. (2005). Mídias das Fontes: o difusor do jornalismo corporativo. Brasília: Casa das Musas.

Schlensiger, P. (1992). Repenser la sociologie du journalisme: Les stratégies de la source d'information et les limites du média-centrisme, Réseaux, 51, 75-98.

Schutz, A. (1967). Collected papers I: The problem of social reality (2a ed.). Amsterdam: Martinus Nihoff / The Hague.

Sousa, J. P. (2000). As noticias e os seus efeitos. Coimbra: Minerva. 
Strauss, A. L., Bucher, R., Ehrlich, D. Sabshin, M. \& Schatzman, L. (1964). Psychiatric Ideologies and Institutions. Glencoe: The Free Press.

Strauss, A. L. (1992). Miroirs et masques: une introduction à l'interactionnisme. París: Métailié.

Traquina, N. (1993). As notícias. En N. Traquina (Ed.). Jornalismo: questões, estórias e "estórias" (pp. 167-176). Lisboa: Vega.

Traquina, N. (2001). O Estudo do Jornalismo no Século XX. São Leopoldo: Ed. Unisinos.

Traquina, N. (2005) Teorias do Jornalismo, porque as notícias são como são. ( $2^{\mathrm{a}}$ Ed.). Florianópolis: Insular.

Travancas, I. S. (1992). O Mundo dos jornalistas. São Paulo: Summus.

Tredan, O. (2009). Do Weblog aos blogs de adolescentes: itinerário de um percurso de pesquisa sobre a prática do blog pelo público jovem. Communicare, 1, (9), 41-59. 